\title{
La Pologne sur le chemin de l'interculturel
}

\section{Anna Żok}

\section{OpenEdition}

\section{Journals}

Édition électronique

URL : http://journals.openedition.org/esp/844

DOI : 10.4000/esp.844

ISSN : 2532-0319

\section{Éditeur}

Centre d'Information sur l'Éducation Bilingue et Plurilingue

\section{Édition imprimée}

Date de publication : 1 juin 2016

Pagination : 75-88

ISSN : 1127-266X

\section{Référence électronique}

Anna Żok, « La Pologne sur le chemin de l'interculturel », Éducation et sociétés plurilingues [En ligne], 40 | 2016, mis en ligne le 28 octobre 2016, consulté le 19 avril 2019. URL : http:// journals.openedition.org/esp/844 ; DOI : 10.4000/esp.844 


\title{
LA POLOGNE SUR LE CHEMIN DE L'INTERCULTUREL
}

\begin{abstract}
Anna ŻoK
La nozione di interculturalità viene oggi declinata secondo nuovi orizzonti: è perciò interessante studiare che cosa si intenda per educazione interculturale in un ex-paese dell'Europa dell'Est, la Polonia. Le ricerche polacche in quest'ambito sono recenti e poco note. Esse permettono di misurare l'influenza del contesto storico e sociale sul modo di affrontare e di trattare le questioni interculturali. Inoltre in questo contributo proponiamo da un lato alcune pratiche educative extra-scolastiche mirate a far scoprire la diversità culturale, linguistica e religiosa spesso ignorata dalla popolazione. Dall'altro, affrontiamo la problematizzazione e la definizione dell'educazione interculturale attraverso $i$ discorsi dei protagonisti diretti. Questo ci porta a mostrare che a livello delle pratiche e dei discorsi la Polonia si spinge a prendere in considerazione l'interculturale senza esservi ancora pienamente entrata.
\end{abstract}

Parole chiave: interculturale; Polonia; storia; diversità culturale; religione; discorso

The notion of interculturality being interrogated in new fields today, it is interesting to see what intercultural education may mean in a formerly Eastern European country. Polish research on the subject is recent and little known, but it allows measuring the influence of the social and historical context on how intercultural questions are approached. We also propose presenting the extra-curricular activities that lead to the discovery of the cultural, linguistic and religious diversity of which the population is often unaware. Theorizing and defining intercultural education here is based on the discourse of the persons we met. It leads us to consider that Poland is on its way to taking interculturality into account without yet having fully entered into it.

Key-words: intercultural; Poland; history; cultural diversity; religion; discourse

\section{INTRODUCTION}

La Pologne, pays considéré comme homogène, vu de l'extérieur, mais aussi et tout autant de l'intérieur, est confrontée à une diversité culturelle, linguistique et religieuse mal connue. La fin du communisme, la construction de la démocratie et, plus récemment, l'intégration européenne ont amené le pays à se repenser et à se pencher sur son hétérogénéité. Devenue l'objet de réflexions, de débats sociaux et de recherches, la diversité se trouve actuellement au cœur des préoccupations polonaises (Żok 2014). De nombreuses actions éducatives, visant à faire connaître les minorités vivant dans le pays, sont aussi entreprises sur le terrain. Dans notre article, nous nous proposons d'apporter un nouveau regard sur la réalité de ce pays ainsi que de 
La Pologne sur le chemin de l'interculturel

A. ŻoK
LA POLOGNE D'HIER ET D'AUJOURD'HUI témoigner des recherches interculturelles dans le domaine de l'éducation et des pratiques éducatives en dehors de l'école. De manière plus générale, nous souhaitons aussi interroger l'avancement de la Pologne sur le chemin de l'interculturel. C'est-à-dire, nous cherchons à voir si la diversité de la population (culturelle, linguistique, religieuse) est prise en compte dans la vie sociale et si oui, comment. Il s'agit d'apporter des éléments de réponse aux questions suivantes: La diversité est-elle reconnue? Cherche-t-on à la faire découvrir et à montrer sa valeur? Par quels moyens?

Avant de poursuivre, il nous parait important de définir les notions d'éducation et d'interculturel. Ainsi, nous entendons par l'éducation l'«[...] intervention humaine volontaire pour diriger dans un sens moral ou social le développement de l'enfant ou de l'adolescent, plus rarement de l'adulte» (Cuq, 2003: 79-80). L'éducation consiste donc en la construction des valeurs et la dimension éthique y occupe une place primordiale. Quant à la notion d'interculturel, elle essaie de rendre compte des relations réciproques entre les cultures et de l'enrichissement mutuel possible, à partir de différences respectives reconnues et comprises: «il s'agit d'instaurer et d'alimenter sans cesse les circulations entre les cultures, les échanges, les passerelles (qu'on emprunte évidemment dans les deux sens), les connexions, les partages» (Abdallah-Pretceille et Porcher 1996: 19-20). Dans cette perspective, la diversité est non seulement reconnue, mais réellement valorisée. Partagée, elle devient une richesse commune.

Le contexte historique et social dans lequel s'inscrit l'éducation interculturelle en Pologne étant très différent du contexte français, il nous parait important d'en donner quelques caractéristiques. A commencer par l'instabilité territoriale, les élargissements et réductions des frontières polonaises, et leur disparition totale. Ainsi, la notion d'identité polonaise a été redéfinie à plusieurs reprises et le territoire national a été habité par différents peuples. En effet, pendant plusieurs siècles la Pologne a été un État multiethnique et multiconfessionnel. Les minorités nationales et ethniques vivant toujours dans le pays en témoignent. Elles sont reconnues au niveau juridique par la loi sur les minorités nationales et ethniques et sur la langue régionale du 6 janvier 2005. La loi reconnait neuf minorités nationales (Biélorusses, Tchèques, Lituaniens, Allemands, Arméniens, Russes, Slovaques, Ukrainiens, Juifs) et quatre minorités ethniques (Karaimes, Lemkos, Roms, Tatars). D'après la loi, il s'agit des groupes de citoyens polonais moins nombreux que le reste de la population, se distinguant par la langue, la culture ou les traditions et dont les ancêtres ont vécu sur le territoire national polonais depuis au moins cent ans. Alors qu'une 
La Pologne sur le chemin de l'interculturel

A. ŻoK minorité nationale s'identifie avec la communauté vivant dans son pays d'origine, ce n'est pas le cas d'une minorité ethnique. La différence entre les minorités nationales et ethniques se situe donc au niveau de la définition juridique de ces groupes. Il faut mentionner aussi les Cachoubes, «la communauté parlant la langue régionale» mais non reconnue officiellement comme une minorité. La reconnaissance d'un groupe comme une minorité est importante dans la mesure où il peut bénéficier de certains droits, notamment en matière de langue, d'éducation et de culture. Malgré la présence des minorités, la population actuelle est plutôt homogène. Les chiffres officiels de l'Institut Central des Statistiques le confirment: l'identité nationale polonaise est déclarée comme unique par 94.8\%, 98.2\% parlent polonais à la maison et la majorité se déclare de religion catholique (entre 86.7 et $95.5 \%$ ). Précisons que ces chiffres ont été obtenus en 2011 lors du dernier recensement de la population de Pologne et que les membres des minorités ont également été questionnés. Alors que, pour la première fois de l'histoire, ce recensement a permis aux enquêtés de reconnaitre leurs appartenances identitaires multiples, la double identité (polonaise et autre) a été déclarée par $2.26 \%$ et l'identité autre que polonaise par $1.55 \%$ de population. Cela montre que les minorités nationales et ethniques, du moins celles déclarant officiellement leur appartenance à une minorité, constituent un pourcentage faible de la population d'aujourd'hui. Malgré cela, elles en font partie intégrante, ce qui explique la nécessité de les prendre en compte dans toute analyse.

Pour mieux comprendre la Pologne, il faut évoquer aussi son histoire mouvementée du $20^{\text {ème }}$ siècle, marquée par la Seconde Guerre mondiale puis le régime communiste. Leur poids pèse toujours sur la société et, notamment, sur les personnes âgées. De l'histoire plus récente, mentionnons l'abandon de l'économie socialiste planifiée au profit du capitalisme, la construction de la démocratie et d'une société libre dans les années 1990, ainsi que l'intégration européenne, clôturée par l'adhésion à l'Union Européenne en 2004. Enfin, l'élection de l'ancien premier ministre polonais Donald Tusk à la présidence du Conseil Européen le $1^{\mathrm{er}}$ décembre 2014 constitue une nouvelle étape de la Pologne dans l'Europe.

Néanmoins, les mentalités ne changent pas du jour au lendemain et la société polonaise semble encore partagée entre le repli nationaliste, c'est-à-dire le renfermement sur soi et la peur de l'autre, et l'ouverture à l'Europe. La montée du nationalisme lors des élections de 2005 ou encore l'importance accordée au fait religieux en sont des exemples. Un besoin constant de reconstruire et de défendre une identité nationale à différents moments de l'histoire explique probablement ces phénomènes modernes. C'est dans ce 
La Pologne sur le chemin de l'interculturel

A. ŻoK

\section{RECHERCHES} EN ÉDUCATION

INTERCULTURELLE EN POLOGNE contexte particulier que sont nées les recherches polonaises sur l’interculturel.

Alors qu'en France, les publications francophones et anglo-saxonnes sur l'éducation interculturelle semblent bien connues, car largement diffusées parmi les chercheurs travaillant sur ce sujet, celles en polonais le sont beaucoup moins, voire pas du tout. D'une part, la langue polonaise ne bénéficie pas de la même présence dans le monde que le français et l'anglais, ce qui limite le nombre de lecteurs potentiels. D'autre part, la littérature polonaise sur l'éducation interculturelle est plus récente (les premières publications datent des années 1990) et donc moins connue. Les publications en langue polonaise sont intéressantes dans la mesure où elles nous permettent de voir comment le contexte historique et social influe sur la conception des thèmes de recherche et sur leur traitement. Il est aussi intéressant d'analyser ce que l'on comprend par «éducation interculturelle» dans un pays de l'ex-bloc communiste confronté à la démocratie et à l'intégration européenne. Après avoir présenté un bref aperçu de la recherche interculturelle en Pologne dans le domaine de l'éducation, nous aborderons l'éducation interculturelle polonaise à travers ses spécificités. Ces dernières seront mises en évidence au niveau des thèmes étudiés. Notre corpus est constitué de textes scientifiques polonais sur l'éducation interculturelle, publiés entre 1995 et 2011 dans des ouvrages collectifs pour la plupart.

Les premières approches interculturelles en éducation émergent en France et, de manière plus générale en Europe, dans les années 1970 et elles sont liées à la scolarisation des enfants d'immigrés. À l'époque, la Pologne fait partie des pays du bloc communiste, tous coupés de l'Europe de l'Ouest par le rideau de fer. La Pologne ne connait pas à l'époque, et elle n'a d'ailleurs jamais connu, l'immigration à la même échelle que la France et d'autres pays européens. Elle n'est donc pas confrontée aux mêmes défis éducatifs. Par ailleurs, à cette période-là, la diversité de la société polonaise est le plus souvent gommée par le gouvernement communiste, parfois exploitée à des fins politiques. D'un côté, le pouvoir communiste proclamant un État-nation polonais parfaitement homogène, de nombreuses actions visent à assimiler les minorités et à nier, à terme, leur présence en Pologne. De l'autre côté, plusieurs campagnes antisémites sont organisées pour diviser la société.

Les questions relevant de l'éducation interculturelle émergent donc en Pologne une vingtaine d'années plus tard qu'en France, dans les années 1990. Qu'est-ce qui se trouve à leur origine? Deux raisons principales semblent expliquer l'intérêt porté à l'époque à l'intercultu- 
La Pologne sur le chemin de l'interculturel

A. ŻoK rel. Dans la Pologne démocratique, on observe d'abord une reconnaissance des minorités nationales et ethniques, ancrées historiquement dans le pays, depuis plusieurs siècles pour certaines, et niées pendant la période communiste. En effet, il est important de rappeler que pendant des siècles, la Pologne a été un État multinational et multiconfessionnel, donc multiculturel. Déjà au Moyen Age, différents peuples vivaient sur le territoire national (Allemands, Juifs, Arméniens, Tatars). Au $16^{\mathrm{eme}}$ siècle, la Pologne et la Lituanie se sont unies donnant naissance à la République des Deux Nations. Ce nouvel État avait un caractère multiethnique (les Polonais représentaient seulement environ $40 \%$ de la population) et se distinguait par sa grande tolérance religieuse. Cette tolérance a épargné à la Pologne les guerres de religion qui ont secoué l'Europe à l'époque. Encore au début du $20^{\mathrm{ème}}$ siècle, la population polonaise était très diversifiée. Face à ce passé multiculturel, comment expliquer l'apparente homogénéité de la société polonaise moderne? Elle peut être considérée comme une conséquence historique et, en particulier, un résultat de la Seconde Guerre mondiale. Ensuite, dans l'intérêt porté à l'interculturel, il y a un désir marqué de faire partie de l'Europe, de s'intégrer avec d'autres pays, un désir d'autant plus fort qu'il nait après presque 45 ans d'isolement. Il s'agit aussi de se préparer à vivre dans un monde globalisé. D'une part, par l'éducation interculturelle, la Pologne semble donc vouloir renouer avec son passé multiculturel et s'interroger sur sa propre diversité (niveau local) et, d'autre part, elle se projette dans l'avenir européen et mondial (niveau global). Ces deux orientations majeures, sous différents thèmes abordés, sont visibles dans plusieurs textes de notre corpus (Lewowicki et alii 2011, Szczurek-Boruta 2000). Retraçons maintenant le développement du champ de recherches en éducation interculturelle. Un événement important dans sa structuration est la première publication dans la collection «Éducation Interculturelle» de l'Université de Silésie en 1992. Cette collection témoigne de l'essor remarquable des recherches polonaises en éducation interculturelle: entre 1992 et 2013, cinquante ouvrages ont été publiés (Ogrodzka-Mazur et al. 2013). Les années 90 sont marquées aussi par les premières conférences sur l'éducation interculturelle, organisées en particulier par les chercheurs de Cieszyn et de Białystok. En 1994, au sein de la Faculté de pédagogie et de psychologie de Białystok, l'Institut de l'Éducation Interculturelle est créé sous la direction de J. Nikitorowicz. Le champ de recherches en éducation interculturelle commence ainsi à se structurer et à acquérir une certaine légitimité au niveau universitaire. Cette dernière se manifeste aussi par l'introduction de la matière intitulée «Éducation interculturelle» dans le programme des études en pédagogie à l'Université de Cieszyn 
La Pologne sur le chemin de l'interculturel

A. ŻoK depuis 2002 (ibid.). Il s'agit toutefois d'initiatives locales dont la portée reste limitée. Par ailleurs, trop récente, avec des théories qui ne sont pas encore solidement établies, l'éducation interculturelle n'est pas à ce jour considérée en tant que spécialité indépendante en pédagogie (Błeszyńska 2010). En fait, les recherches polonaises dans ce domaine se situent souvent à la croisée de la pédagogie et d'autres disciplines, comme l'ethnologie, l'histoire, la linguistique, l'anthropologie culturelle, la psychologie et la sociologie. Cette interdisciplinarité est visible non seulement au niveau théorique, mais aussi au niveau pratique, dans des projets éducatifs (Lewowicki et alii. 2011).

Le champ de recherches en éducation interculturelle émergeant en Pologne plus tard que dans les pays occidentaux, certaines influences étrangères sont visibles au niveau théorique. Dans les textes scientifiques que nous avons pu consulter, nous trouvons des références notamment aux travaux des chercheurs français et allemands. Par ailleurs, de nombreux exemples de l'éducation multiculturelle et interculturelle dans le monde sont décrits. Les chercheurs polonais soulignent cependant que les solutions éducatives mises en place dans différents pays ne conviennent pas nécessairement à la Pologne où certaines minorités vivent depuis des siècles. Quel est donc leur intérêt? C'est avant tout de montrer que chaque société devrait constamment chercher des réponses à sa propre diversité culturelle. En effet, s’il n'existe pas de modèle universel de l'éducation interculturelle, des rapprochements, des transversalités entre les divers contextes se présentent: différences et ressemblances sont constitutives de recherches sur l'interculturel. Les expériences des autres pays face à la diversité aident donc à comprendre quels sont les aspects importants à prendre en compte (Szczurek-Boruta 2000).

La conception de l'éducation interculturelle, telle qu'elle apparait dans les textes scientifiques polonais, ressemble partiellement à la conception française, notamment à travers les notions mobilisées. Les différences sont toutefois perceptibles, en particulier au niveau des thèmes abordés. Dans la suite, nous allons présenter quelques thèmes spécifiques à l'approche polonaise des problématiques interculturelles en éducation.

Il est important de rappeler que jusqu'en 1989, la problématique du multiculturalisme était quasiment inexistante dans le discours public polonais. Après la chute du régime communiste, cette problématique commence à être abordée. Dans les travaux scientifiques, deux thématiques majeures peuvent être distinguées. D'un côté, on s'intéresse aux immigrés, aux étrangers et aux réfugiés, et notamment, à leur adaptation culturelle. De l'autre côté, on étudie les minorités nationales et ethniques vivant en Pologne. Dans les textes de notre corpus, la secon- 
La Pologne sur le chemin de l'interculturel

A. ŻoK de thématique, celle liée aux minorités, est largement dominante.

Dans les années 1990, le multiculturalisme devient un sujet d'actualité compte tenu de la perspective de l'adhésion de la Pologne à l'Union Européenne. On s'interroge alors sur les identités européenne et polonaise, les conditions de l'ouverture à l'Autre et la communication interculturelle. De nombreux ouvrages sont publiés au début des années 2000, quand l'intégration européenne se concrétise. On souligne alors la nécessité d'élaborer un modèle (ou des modèles) de l'éducation interculturelle en Pologne (Lewowicki 2002).

Les réflexions polonaises sur le multiculturalisme et la tolérance se caractérisent en particulier par des références fréquentes à l'histoire. On parle notamment du passé multiculturel du pays. Précisons qu'à la veille de la Seconde Guerre mondiale, les minorités constituaient 30\% de la population. Parmi les plus nombreuses, nous pouvons mentionner, dans l'ordre décroissant, des Ukrainiens, des Juifs, des Biélorusses et des Allemands. Le défi moderne de la diversité semble donc s'inscrire dans la continuité de la nation polonaise.

Par ailleurs, les recherches interculturelles en Pologne présentent des similitudes avec celles menées dans d'autres anciens pays communistes. Il s'agit notamment des thèmes communs, parmi lesquels nous pouvons mentionner: les minorités indigènes, les cultures des régions frontalières, la reconstruction du passé multiculturel, l'amélioration des relations interethniques. En conséquence, les groupes de recherches s'intéressent souvent à des régions spécifiques et, notamment, celles situées près des frontières, où ces phénomènes peuvent être étudiés (Błeszyńska 2010). Ainsi, en Pologne, à l'exception de Varsovie, les universités spécialisées dans les problématiques interculturelles se trouvent toutes dans les régions frontalières.

La problématique de l'éducation interculturelle en Pologne englobe non seulement les rapports entre la majorité et les minorités nationales et ethniques, mais elle s'intéresse aussi à d'autres groupes considérés comme minoritaires. Il s'agit notamment des personnes handicapées et des sourds. En prenant en compte ces minorités, les chercheurs soulignent que l'éducation interculturelle devrait aider à accepter l'altérité au sens large: culturelle, religieuse, linguistique, physique.

Une autre spécificité des textes polonais sur l'éducation interculturelle est la prise en compte de la thématique religieuse. En plus du côté humaniste de l'éducation interculturelle, son côté religieux est mis en lumière. En effet, certains chercheurs soulignent que cette éducation relève de l'éthique chrétienne. Il est notamment question de l'amour du prochain et d'un grand respect pour la dignité l'être humain, c'està-dire des valeurs reconnues dans l'axiologie chrétienne auxquelles les approches interculturelles se réfèrent également. Par ailleurs, l'accent 
La Pologne sur le chemin de l'interculturel

A. ŻoK est mis sur l'éducation religieuse et le développement de la tolérance pour d'autres religions dans le cadre de l'éducation interculturelle. Plus précisément, cette dernière devrait viser à promouvoir le dialogue interreligieux, une véritable rencontre qui dépasse la tolérance et la coexistence pacifique de différentes confessions. Les chercheurs insistent également sur le rôle des églises présentes en Pologne dans la promotion du dialogue entre différentes religions. Ils n'abordent cependant pas la question de l'antisémitisme. Un travail personnel, au niveau de l'identité religieuse, est également nécessaire: afin d'entreprendre le dialogue avec les représentants d'autres confessions, l'individu doit connaître et comprendre sa propre religion. Même si cette intention peut paraitre théorique, elle est d'actualité dans le contexte polonais où le catholicisme occupe une place importante dans la vie sociale. Les entretiens avec les acteurs de terrain montrent que les jeunes polonais connaissent mal leur propre religion.

Il est intéressant de voir aussi quelle est la place accordée aux langues dans l'éducation interculturelle polonaise. Alors qu'en France, l'éducation interculturelle est historiquement liée à l'enseignement des langues d'origine et du français aux enfants migrants, les questions linguistiques se présentent différemment en Pologne. De manière générale, elles sont peu évoquées dans les textes analysés. Si elles sont abordées, c'est pour insister sur l'importance de l'apprentissage des langues étrangères par les Polonais. La compétence linguistique est présentée comme indispensable dans la rencontre interculturelle. Elle permet également de profiter pleinement des possibilités de mobilité offertes par la construction européenne (Szczurek-Boruta 2000). Quant aux langues des minorités nationales et ethniques vivant en Pologne, elles sont rarement évoquées, sauf quand il s'agit de présenter l'enseignement d'une langue donnée. Il n'y a pas non plus de fil conducteur dans la réflexion sur les langues minoritaires. Le plus souvent, on souligne la nécessité de les respecter pour éviter une domination linguistique et de les transmettre au sein des familles. Plus rarement, on préconise leur enseignement aux enfants issus des minorités dans le cadre de l'éducation interculturelle. On ne présente toutefois pas les langues minoritaires comme une richesse qui pourrait être découverte par d'autres. Enfin, certains chercheurs s'interrogent sur la place de l'espéranto dans l'éducation interculturelle. Partagée par les représentants de différentes cultures et origines, cette langue peut favoriser la compréhension et la coopération au-delà des frontières (Grzybowski 2008). Même si l'utilité pratique de l'espéranto demeure l'objet de débats, l'idée d'une langue commune semble intéressante pour l'éducation interculturelle (ibid.).

Le tour de la question proposé ci-dessus n'a pas la prétention de 
La Pologne sur le chemin de l'interculturel

A. ŻoK

PRATIQUES

ÉDUCATIVES EN

DEHORS DE

L'ÉCOLE: VERS

L'INTERCULTUREL constituer un véritable état des lieux des recherches en Pologne sur l'interculturel. Un panorama plus systématique des articles de revues, des colloques récents et des cours à l'université serait sans doute à réaliser. Comme nous avons montré de manière synthétique, les ouvrages publiés en Pologne alertent de façon très nette sur quelques spécificités liées au contexte historique et social de leur émergence.

En plus de recherches menées en Pologne sur l'éducation interculturelle, de nombreux projets éducatifs sont actuellement mis en place sur le terrain. Dans le cadre de notre recherche doctorale, nous nous sommes intéressée à l'éducation pratiquée en dehors de l'école (dans des centres culturels, associations, etc.) et nous avons interrogé leurs différents acteurs. Les discours recueillis permettent de savoir non seulement comment l'éducation interculturelle est mise en œuvre dans diverses institutions, mais aussi comment elle est problématisée par ceux qui la pratiquent ou, tout au moins, pensent le faire. De manière plus générale, les discours produits sont des indicateurs de l'avancement de la Pologne sur le chemin de l'interculturel.

Des entretiens semi-directifs ont été effectués en 2011 dans plusieurs villes polonaises situées dans deux régions: en Poméranie (Gdańsk, Gdynia, Koszalin, Słupsk) et en Silésie (Wrocław). Ces régions sont particulièrement intéressantes pour aborder des questions interculturelles, car elles sont historiquement marquées par des contacts entre différents peuples. Les interviews ont été réalisées auprès de personnes concernées par l'interculturel, mais de manière inégale et pour des raisons diverses: des professionnels de l'éducation extra-scolaire, des présidents des associations minoritaires, des membres des minorités. Leurs actions sur le terrain de l'éducation extra-scolaire sont variables. Les uns mettent en place l'éducation interculturelle ou s'engagent dans divers projets. Les autres ne montrent aucun engagement, ou très peu, dans la pratique de l'éducation interculturelle. Au total, vingt-six personnes ont été interviewées. Notre travail relevant de l'approche qualitative, nous avons choisi de construire un échantillon limité mais diversifié. Les résultats obtenus ne peuvent donc pas être généralisés mais ils sont révélateurs de certains faits et tendances.

Les discours recueillis permettent d'abord de dresser le cadre général de l'éducation interculturelle extra-scolaire. Cependant, il ne s'agit nullement de dresser ici un état des lieux complet, notre objectif étant de présenter les initiatives entreprises par nos seuls informateurs. Plus précisément, leurs discours apportent des éléments de réponse aux questions suivantes: Quels sont les besoins et les objectifs éducatifs? Quelles sont les pratiques éducatives?

En ce qui concerne le public visé par l'éducation interculturelle, deux 
La Pologne sur le chemin de l'interculturel

A. ŻoK types de réponse peuvent être distingués: le groupe d'âge à éduquer et le type de population. Compte tenu des groupes mentionnés le plus souvent par nos informateurs et de la façon dont ils le font, nous pouvons énumérer dans l'ordre d'importance: les enfants, les jeunes, les adultes, les personnes âgées. L'évocation de ces groupes structure l'ensemble des discours, qu'il s'agisse des projets existants ou futurs. L'éducation des enfants apparaît comme une priorité et les discours produits témoignent d'une variété de projets déjà mis en place auprès de ce public.

Quant au type de la population à éduquer, il est moins souvent mentionné. De manière générale, les discours - qu’ils soient produits par les personnes issues des minorités ou de la majorité - montrent que l'éducation de la majorité est une nécessité, comme en témoigne cet extrait d'entretien: «Mais ce spectateur qui pourrait venir à un tel festival se déplacer consacrer son temps libre / il faut d'abord le préparer / parmi cette majorité / et l'éduquen».

Parmi les objectifs les plus importants qui ne relèvent pas du contexte et qui pourraient probablement être définis dans d'autres pays européens, nous pouvons mentionner:

- faire découvrir la diversité et apprendre à l'exploiter comme une richesse;

- promouvoir les attitudes de tolérance et d'ouverture;

- dépasser les stéréotypes, changer les attitudes face à l'Autre.

Alors que ces objectifs semblent être des universaux et applicables dans d'autres contextes, certains discours témoignent aussi d'objectifs plus spécifiques au contexte polonais. Parmi ces derniers, nous avons répertorié:

- dépasser le poids de l'histoire, oublier les guerres et affrontements avec les pays voisins: «L'histoire est parfois pénible / l'histoire est parfois pénible et hmhm / elle blesse parfois / parfois elle rappelle euh / des souvenirs difficiles»;

- montrer l'ancrage historique des minorités en Pologne et le passé multiculturel du pays: «une mosaïque / une tentative de montrer cette image multiculturelle a ses avantages / par rapport à l'histoire de la Pologne»;

- se préparer à accueillir des immigrés dans l'avenir: «il faut ouvrir la société aussi / car euh / si les immigrants viennent ici [...] et s’ils sont rejetés / s'il y a un ostracisme social / ils créeront alors des groupes fermés».

Les premiers objectifs mentionnés tendraient à prouver l'existence de certains universaux éducatifs, relevant de l'humanisme et des valeurs partagées par plusieurs sociétés. Cependant, au-delà des universaux, il semble crucial de tenir compte du contexte dans lequel les actions éducatives sont réalisées. Un pays comme la Pologne montre qu'il ne suffit pas de se pencher sur la société actuelle, le poids de l'histoire devant être pris en considération. Les discours recueillis permettent de 
La Pologne sur le chemin de l'interculturel

A. ŻoK voir ce qui est général et ce qui est particulier dans les approches interculturelles en Pologne.

Concernant les pratiques éducatives en dehors de l'école, elles se caractérisent par une grande diversité dont témoignent les discours produits. La forme des activités varie notamment en fonction de l'âge du public. De manière générale, pour les enfants et les jeunes, des activités plus ludiques et interactives sont choisies. Elles prennent souvent la forme d'ateliers. La culture se pratique ainsi en actes, dans l'action et non par le seul biais de discours sur elle. Le thème de la rencontre («rencontrer les gens») émerge de la vision qu'ont nos informateurs d'une éducation interculturelle. Les rencontres avec différentes minorités sont ainsi organisées pour tout public. De manière plus spécifique, aux adultes, on propose aussi des conférences, des festivals et des concerts. Bien qu'elles soient différentes les unes des autres, ces activités visent le même but: montrer la diversité culturelle de la société polonaise actuelle ainsi que favoriser les attitudes d'ouverture et de tolérance.

S'agissant des contenus, trois tendances générales peuvent être distinguées. Premièrement, nous remarquons qu'en plus de la culture, la religion occupe une grande place dans les projets éducatifs, notamment ceux adressés aux enfants et aux jeunes («projets multireligieux», «rencontres interreligieuses»). Cette importance accordée à l'aspect religieux a déjà été constatée dans les textes d'experts polonais. L'Église catholique a joué un rôle crucial à différents moments de l'histoire et le poids de cette institution se fait toujours sentir au sein de la société actuelle. La société polonaise semble donc avoir besoin d'une ouverture à d'autres religions et d'une éducation à la tolérance envers elles.

Deuxièmement, les organisateurs des projets éducatifs essaient de montrer non seulement ce qui est différent dans diverses cultures ou religions mais aussi ce qui est commun: «et ainsi les enfants apprenaient / ils apprenaient hmhm ici il y a / nous avons ça de commun / et ça aussi nous l'avons de commun / mais ici ils prient dans cette langue / n'est-ce pas? [...] Ici en hébreu / ici en slave liturgique». A travers ces discours, nous retrouvons les fondements de l'éducation interculturelle, les rapports entre l'Autre et le Même étant au cœur de ses préoccupations: «Plus qu'un discours sur l'Autre, il s'agirait de promouvoir un discours sur les rapports réciproques entre Moi et l'Autre, discours qui constituerait l'essence même du discours interculturel» (Abdallah-Pretceille, 1996: 75).

Troisièmement, les interviewés constatent que la rencontre de l'Autre commence souvent par la découverte de son quotidien. Ainsi, dans le cadre des rencontres organisées avec les minorités ou les étrangers, ce 
La Pologne sur le chemin de l'interculturel

A. ŻoK sujet est abordé en premier. Les discours montrent que les contenus relatifs à la vie de tous les jours sont considérés comme la base. D'autres sujets, plus complexes (comme la littérature, l'histoire, les valeurs) peuvent être abordés par la suite.

Au-delà du cadre général de l'éducation interculturelle, à travers les discours, nous pouvons voir comment l'éducation interculturelle est problématisée par une population polonaise particulière (professionnels de l'éducation extra-scolaire, représentants des minorités, fonctionnaires d'État). De manière plus générale, ces discours indiquent où se trouve la Pologne sur le chemin de l'interculturel.

La problématisation de l'éducation interculturelle a déjà été mentionnée à plusieurs reprises: nous avons montré la prise en compte du contexte historique et social par nos informateurs, les besoins et les objectifs formulés, les pratiques orientées vers les moyens de l'action et de la rencontre. Par ailleurs, les discours recueillis montrent que la problématisation de l'éducation interculturelle, même si elle répond bien à des exigences éthiques fortes et incontournables, reste limitée. Nos informateurs ont effectivement une vision réductrice de l'interculturel. Nous pouvons distinguer deux «pas» dans leur démarche qu'ils ne franchissent jamais.

Les discours témoignent d'une démarche qui ne va que dans un seul sens. On s'intéresse à l'Autre - et c'est déjà bien compte tenu du contexte historique et social polonais - mais sans revenir vers soi par la suite. C'est pourquoi, il ne s'agit pas ici d'une véritable démarche interculturelle. Cette dernière nécessite un retour vers soi, un soi modifié par la rencontre avec l'Autre: «[...] la confrontation des écarts permet de mieux connaitre et comprendre ses propres spécificités, de prendre la mesure de ce qu'on est; elle permet dans la rencontre avec l'autre de se quitter soi-même pour se retrouver autrement» (Delamotte-Legrand 2006: 41). L'effort du «décentrage» est donc essentiel dans la démarche interculturelle. Celle-ci ne s'accomplit que quand la rencontre avec l'Autre provoque une transformation de soi. Nos acteurs ne vont pas jusqu'à ce point ni dans la problématisation de l'éducation ni dans leurs actions.

La dimension créative de l'interculturel n'est jamais évoquée par nos informateurs. La création d'une réalité nouvelle est cependant un des aspects essentiels des approches interculturelles, ce qui est mis en évidence aussi bien par les chercheurs français que polonais. Il s'agit de l'émergence de formes ou de pratiques inédites, métissées, imprégnées des influences variées. Ces pratiques partagées et assumées, qui vont au-delà de ce qu'on est soi et de ce qu'est l'Autre, témoignent d'un processus interculturel. Tant que cet aspect créatif n'est pas envisagé comme un possible résultat de la rencontre entre cultures différentes, 
La Pologne sur le chemin de l'interculturel

A. ŻoK

la conceptualisation de la notion d'interculturel en reste à une juxtaposition des cultures. L'ambition éducative demeure donc au premier niveau de la tolérance, au mieux à celui de la curiosité et de l'intérêt pour ce qui n'est pas soi.

L'absence de ces deux aspects est significative: elle montre qu'au niveau des pratiques, la Pologne est sur le chemin de l'interculturel. Le contexte actuel y est d'ailleurs favorable, l'intégration européenne invitant à la prise en compte de la diversité. Ainsi, cette dernière commence à être reconnue, on cherche à la faire découvrir et à la valoriser. Mais il reste encore beaucoup à faire pour sortir de cet état «préinterculturel» au profit d'un réel processus interculturel.

\section{CONCLUSION}

\section{BIBLIOGRAPHIE}

Nous pouvons retenir, au terme de cet article, les spécificités de l'approche polonaise des problématiques interculturelles en éducation: les références au passé multiculturel du pays, la prise en compte de différents groupes minoritaires, la place réduite réservée aux langues, la thématique religieuse. Il est intéressant de voir que cette dernière s'inscrit dans l'interculturel. L'interculturel s'oppose en cela à d'autres courants qui intègrent aussi la religion, mais l'exploitent de manière différente, comme par exemple les nationalistes polonais. Néanmoins, cette approche polonaise, tout en tenant compte des particularités dues à son terrain d'émergence, intègre certains traits caractéristiques d'autres approches et d'autres contextes. Cela montre que la diversité est une réalité universelle et sa gestion l'enjeu commun des sociétés modernes.

ABDALLAH-PRETCEILLE M. 1996. Vers une pédagogie interculturelle, Paris, Anthropos, 3e édition.

ABDALLAH-PRETCEILLE M., PORCHER L. 1996. Éducation et communication interculturelle, Paris, PUF.

BŁESZYŃSKA K. M. 2010. Intercultural Education in PostCommunist Countries, pp. 69-82 in C. A. GRANT, A. PORTERA (eds.), Intercultural and Multicultural Education: Enhancing Global Interconnectioness, New York, Routledge.

CUQJJ-P. (dir.) 2003. Dictionnaire de didactique du français langue étrangère et seconde, Paris, Clé International, SEJER.

DELAMOTTE-LEGRAND R. 2006. Écarts langagiers: entre hétérogénéité, diversité et altérité, pp. 37-57 in C. HÉLOT et al., Écarts de langue, écarts de culture, Berlin, Bern, Peter Lang.

GRZYBOWSKI P. P. 2008. Edukacja miedzykulturowa - przewodnik. Pojeccia, literatura, adresy (Éducation interculturelle - guide. Notions, littérature, adresses), Kraków, Oficyna Wydawnicza Impuls.

LEWOWICKI T. 2002. W poszukiwaniu modelu edukacji między- 
La Pologne sur le chemin de l'interculturel

A. ŻoK kulturowej (A la recheche d'un modèle de l'éducation interculturelle), pp. 15-32 in T. LEWOWICKI et al., Edukacja wobec ładu globalnego (L'éducation face à l'ordre global), Warszawa, Wydawnictwo Akademickie Żak.

LEWOWICKI T., OGRODZKA-MAZUR E., SZCZUREKBORUTA A. (red.) 2011. Edukacja międzykulturowa-dokonania, problemy, perspektywy (L'éducation interculturelle - réalisations, problèmes, perspectives), Toruń, Wydawnictwo Adam Marszałek.

OGRODZKA-MAZUR E., SZCZUREK-BORUTA A. 2013. Działalność naukowo-badawcza Zakładu Pedagogiki Ogólnej Uniwersytetu Śląskiego na Wydziale Etnologii i Nauk o Edukacji w latach 1989-2012. Wokół cieszyńskiej szkoły badań pogranicza (Les activités d'enseignement et de recherche de l'Institut de Pédagogie Générale de l'Université de Silésie au sein de la Faculté d'ethnologie et des sciences de l'éducation de 1989 à 2012. Autour de l'école de recherches de Cieszyn sur la zone frontalière), pp. 219-232 in Edukacja Międzykulturowa (Éducation Interculturelle), nº 2.

SZCZUREK-BORUTA A. 2000. Szkoły na Podbeskidziu wobec wyzwań edukacji międzykulturowej (Les écoles de Podbeskidzie face aux défis de l'éducation interculturelle), pp. 129-144 in T. LEWOWICKI, E. OGRODZKA-MAZUR, A. SZCZUREK-BORUTA (red.), Edukacja międzykulturowa w Polsce i na świecie (Éducation interculturelle en Pologne et dans le monde), Cieszyn, Filia Uniwersytetu Śląskiego, Warszawa, Wyžsza Szkoła Pedagogiczna ZNP.

ŻOK A. 2014. Éducation interculturelle: textes, discours, pratiques déclarées. Contexte de la Pologne actuelle, Thèse de doctorat en sciences du langage, Regine Delamotte-Legrand (dir.), Université de Rouen. 